\title{
Innenarchitektur der Zellkraftwerke - Membranfalten in Hochauflösung
}

\author{
TILL STEPHAN, PETER ILGEN, STEFAN JAKOBS \\ UNIVERSITÄTSMEDIZIN GÖTTINGEN \& MAX-PLANCK-INSTITUT \\ FÜR BIOPHYSIKALISCHE CHEMIE
}

\section{Mitochondria are essential cellular organelles, which supply eukaryo- tic cells with the universal energy carrier adenosine triphosphate. These organelles feature a unique double-membrane architecture, which is formed by a smooth outer membrane and a highly folded inner membrane. Harnessing super-resolution light and electron microscopy, we investigate the role of MICOS, a large mitochondrial protein complex, in determining the complex folding of the inner membrane.}

DOI: $10.1007 / \mathrm{s} 12268-021-1543-2$

(C) Die Autoren 2021
Erst das Zusammenspiel einer Vielzahl subzellulärer Prozesse ermöglicht komplexe Funktionen wie das Schlagen des Herzens oder die Weiterleitung von Nervenimpulsen. Der wichtigste chemische Brennstoff zum Antrieb solcher Prozesse ist das Molekül Adenosintriphosphat (ATP), welches den Zellen als universeller und sofort verfügbarer Energieüberträger dient. Der größte Teil des verfügbaren ATPs wird in den Mitochondrien, kleinen Zellorganellen mit einem Durchmesser von etwa 200-800 nm, erzeugt. Daher werden diese Organellen gerne als „Kraftwerke der Zelle“ bezeichnet.

Das Geheimnis ihrer besonderen Funktion der Energiegewinnung liegt im einzigartigen strukturellen Aufbau der Mitochondrien. Sie besitzen eine glatte äußere Membran, die eine zweite, stark eingefaltete innere Membran umschließt. Lamellenartige Einstülpungen der Innenmembran werden Cristae genannt und repräsentieren mitochondriale Subkompartimente (Abb. 1). Im Gegensatz zur inneren Grenzflächenmembran ist die

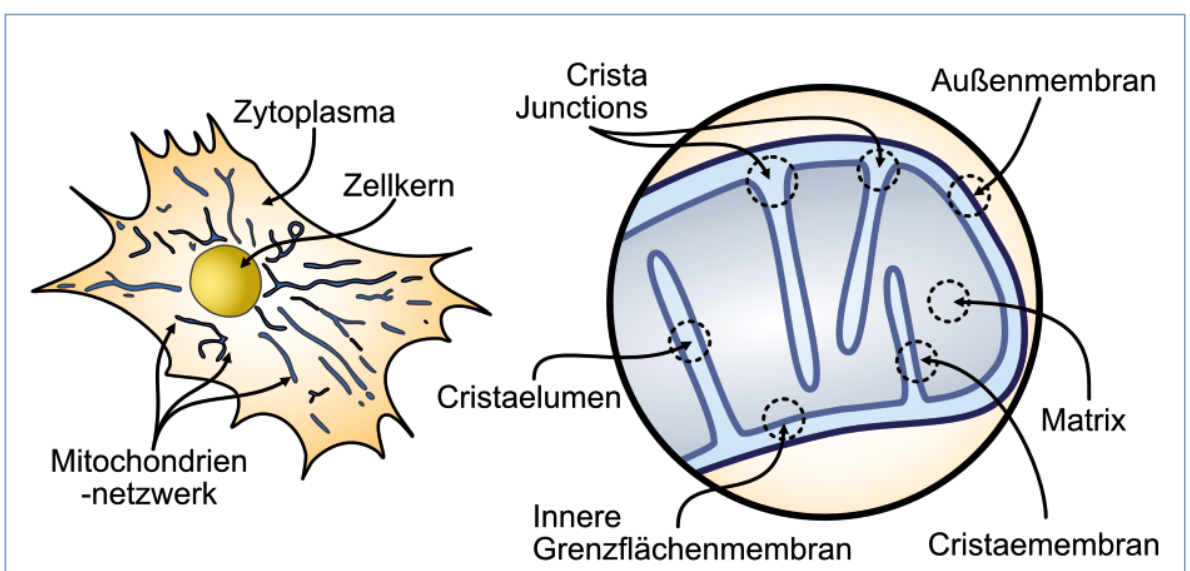

$\Delta$ Abb. 1: Schematische Darstellung des mitochondrialen Netzwerks in einer Zelle und der mitochondrialen Membranarchitektur.
Cristaemembran (Abb. 1) mit den Atmungskettenproteinkomplexen angereichert. Um die erhöhte Konzentration dieser Proteinkomplexe in den Cristae zu gewährleisten, sind Cristaemembran und innere Grenzflächenmembran lediglich durch kleine, runde bis schlitzförmige Öffnungen mit einem Durchmesser von nur 20-30 nm verbunden (Abb. 2), die als Crista Junctions bezeichnet werden.

Die Atmungskettenkomplexe bewerkstelligen die Bildung von ATP. Hierzu werden über mehrere Zwischenschritte zunächst Protonen (positiv geladene Wasserstoffatome) aus der Matrix, dem Innenraum des Mitochondriums, in das Cristaelumen transportiert. Dabei wird ein Gradient über die Membran erzeugt. Die mitochondriale $\mathrm{F}_{1} \mathrm{~F}_{0}$ ATPase, ein in die Cristaemembran integrierter Proteinkomplex, baut diesen Protonengradienten wieder ab und nutzt die dabei freiwerdende Energie ähnlich einer Turbine zur Erzeugung von ATP.

\section{Untersuchung der mitochondrialen} Struktur durch Mikroskopie

Obwohl der besondere Aufbau der Mitochondrien bereits vor etwa 70 Jahren entdeckt wurde, ist bis heute umstritten, wie die komplizierte Cristaearchitektur genau entsteht. Gesichert ist, dass dabei zahlreiche unterschiedliche Proteine beteiligt sind, die die Fähigkeit besitzen, Membranen zu binden und aktiv zu verformen [1]. Die genaue Aufklärung der Entstehung von Cristae ist anspruchsvoll, da Mitochondrien dynamisch sind und eine sehr geringe Größe aufweisen. Tatsächlich ermöglicht die klassische Transmissionselektronenmikroskopie (TEM) eine präzise Analyse der Mitochondrienstruktur (Abb. 2). Da hierbei allerdings die Elektronen die Probe durchstrahlen müssen, ist es notwendig, diese zuvor in sehr dünne Scheiben zu schneiden. Größere Volumen lassen sich besser mit der Focused-Ion-Beam-ScanningElektronen-Mikroskopie (FIB-SEM) analysieren, bei der abwechselnd ein Ionenstrahl eine dünne Schicht der Probe abträgt und die darunterliegende Oberfläche anschließend 


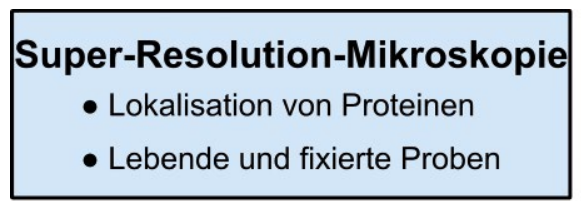

\section{Elektronenmikroskopie}

- Visualisierung von Membranen

- Fixierte Proben
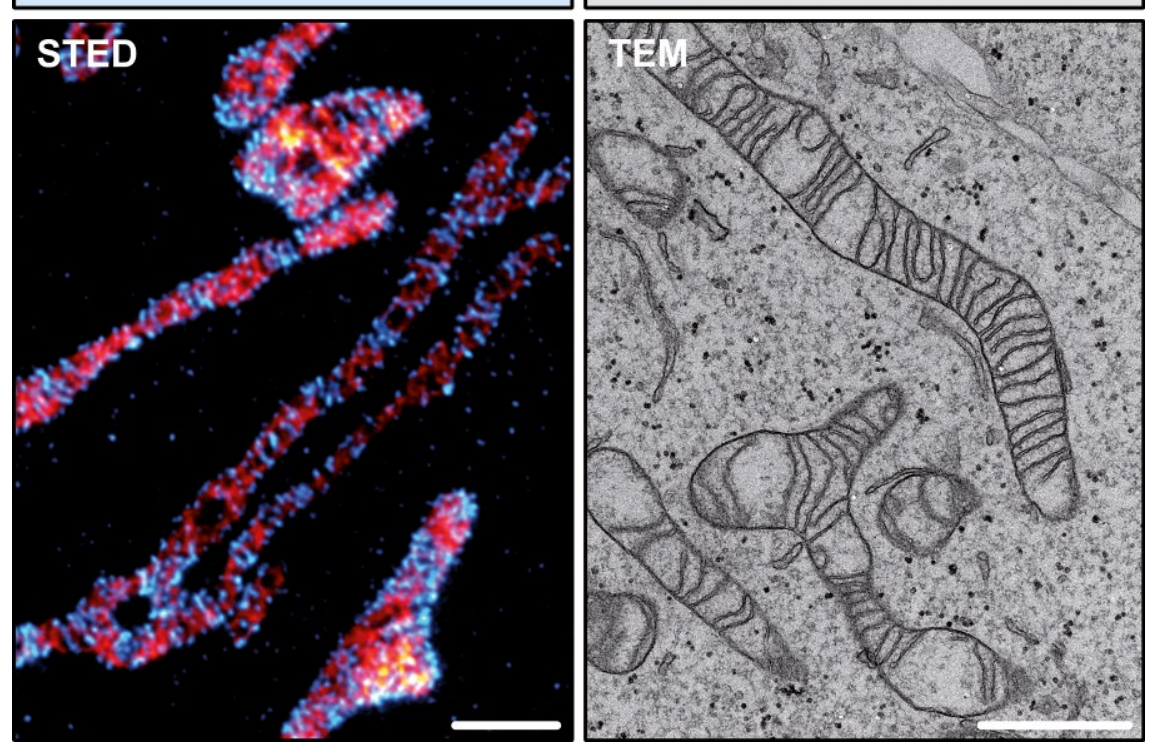

A Abb. 2: Mikroskopie von Mitochondrien. A, Super-Resolution-Mikroskopie von Mitochondrien. Ausschnitt einer STED-Mikroskopieaufnahme. Markiert wurde das Protein MIC60 an den Crista Junctions (blau) und das Protein ATPB in der Cristaemembran (rot). B, TEM-Aufnahme von Mitochondrien. Größenbalken: $1 \mu \mathrm{m}$.

durch das Scannen mit einem Elektronenstrahl abgebildet wird. Obwohl beide Techniken die Cristae zuverlässig visualisieren, haben sie gemeinsam, dass sie nur auf fixierten, also toten Zellen angewendet werden können. Zudem lassen sich mit ihnen Proteine innerhalb der Zelle nur eingeschränkt lokalisieren.

Die Fluoreszenz-Lichtmikroskopie bietet hier Vorteile, da sie statt Elektronen sichtbares Licht verwendet. So können nicht nur gezielt einzelne Proteinspezies mit einem fluoreszierenden Farbstoff markiert und dann in der Zelle lokalisiert (Abb. 2), sondern auch lebende Zellen untersucht werden. Allerdings ist das Auflösungsvermögen von Lichtmikroskopen im Vergleich zu Elektronenmikroskopen stark begrenzt. Der Wellencharakter des Lichts ist dafür verantwortlich, dass die Linsen eines Lichtmikroskops das Licht nicht beliebig genau fokussieren können, was das Auflösungsvermögen konventioneller Fluoreszenzmikroskope in der Praxis auf etwa 200 nm begrenzt. Da dieser Wert im Bereich des Durchmessers eines Mitochondriums liegt, können konventionelle Lichtmikroskope die submitochondriale Verteilung von Proteinen kaum analysieren.

\section{Super-Resolution-Mikroskopie}

Die Entwicklung hochauflösender SuperResolution-Mikroskope in den 2000er-Jahren ermöglichte es, das fundamentale Auflösungsproblem zu lösen; diese Entwicklung wurde 2014 mit dem Nobelpreis für Chemie gewürdigt. Heute stehen Biowissenschaftlern zahlreiche unterschiedliche beugungsunbegrenzte Lichtmikroskope zur Verfügung. Der „Trick“ zur Überwindung der Auflösungsgrenze besteht darin, dass diese Mikroskopieverfahren unterschiedliche Zustände der fluoreszierenden Moleküle ausnutzen, um benachbarte Fluorophore zeitlich oder räumlich voneinander getrennt aufnehmen und dadurch doch unterscheiden zu können (Abb. 2, [2]). STED-Super-Resolution-Mikroskopie erlaubt es auf diese Weise beispielsweise, die dynamische Bewegung einzelner Cristae innerhalb von Mitochondrien mit einer Auflösung von ca. $50 \mathrm{~nm}$ zu verfolgen und so völlig neue Einblicke in die Dynamik des Organells zu erhalten [3, 4]. MINFLUX-Nanoskopie, eine neuere darauf aufbauende Technik, ermöglicht inzwischen sogar eine dreidimensionale Lokalisation von Molekülen mit einer Präzision im einstelligen Nanometerbereich $[5,6]$.

\section{Der MICOS-Komplex}

Das mitochondriale Kontakstellen- und Cristae-Organisations-System, kurz MICOS (mitochondrial contact site and cristae organizing system) ist ein großer Proteinkomplex in der inneren Membran, der eine zentrale Rolle bei der Ausformung der Cristae spielt [7]. Er besteht aus mindestens sieben unterschiedlichen Proteinen, die in zwei Teilkomplexen organisiert sind. Sie werden gemäß ihrer beiden Kernproteine MIC60- bzw. MIC10-Teilkomplex genannt. Obwohl die Teilkomplexe bereits 2011 entdeckt wurden, blieb ihre präzise Rolle bei der Cristaebildung zunächst unklar.

Um die Funktion der unterschiedlichen MICOS-Teilkomplexe detailliert zu analysieren, haben wir in einer Studie die Synthese der sieben MICOS-Proteine mithilfe der Genschere CRISPR-Cas9 systematisch in kultivierten menschlichen Zellen unterbunden [8]. Der Verlust der MIC60-Proteine löst in humanen Zellen den Abbau des vollständigen MICOS-Komplexes aus. Dies verhindert die Bildung von Crista Junctions und führt so letztlich zum Ablösen der Cristae von der inneren Grenzflächenmembran. Durch gezieltes Entfernen des MIC10-Proteins ist es hingegen möglich, lediglich einen Abbau der MIC10-Teilkomplexe herbeizuführen, während die MIC60-Teilkomplexe in den Mitochondrien stabil zurückbleiben. MIC10-defiziente Mitochondrien weisen eine stark veränderte Cristaearchitektur auf und bilden große, flachliegende Cristae, die parallel zur Außenmembran verlaufen (Abb. 3A). Aufgrund der Anwesenheit der MIC60-Teilkomplexe sind diese allerdings weiterhin durch zahlreiche Crista Junctions mit der inneren Grenzflächenmembran verbunden (Abb. 3A). Untersuchungen mittels Elektronentomographie und MINFLUX-Nanoskopie zeigten übereinstimmend, dass die Crista Junctions in Abwesenheit des MIC10-Teilkomplexes allerdings eine abberrante, eher schienenartige Verteilung aufweisen (Abb. 3A, [8]).

\section{Cristaebildung auf Knopfdruck}

Die Tatsache, dass beide MICOS-Teilkomplexe unterschiedliche Funktionen ausüben, ermöglichte es, das Zusammenspiel beider Teilkomplexe bei der Bildung der lamellenförmigen Cristae genauer zu analysieren. Dazu stellten wir die Produktion von MIC10 in den vormals MIC10-defizienten Zellen 
Hier steht eine Anzeige.

包 Springer 


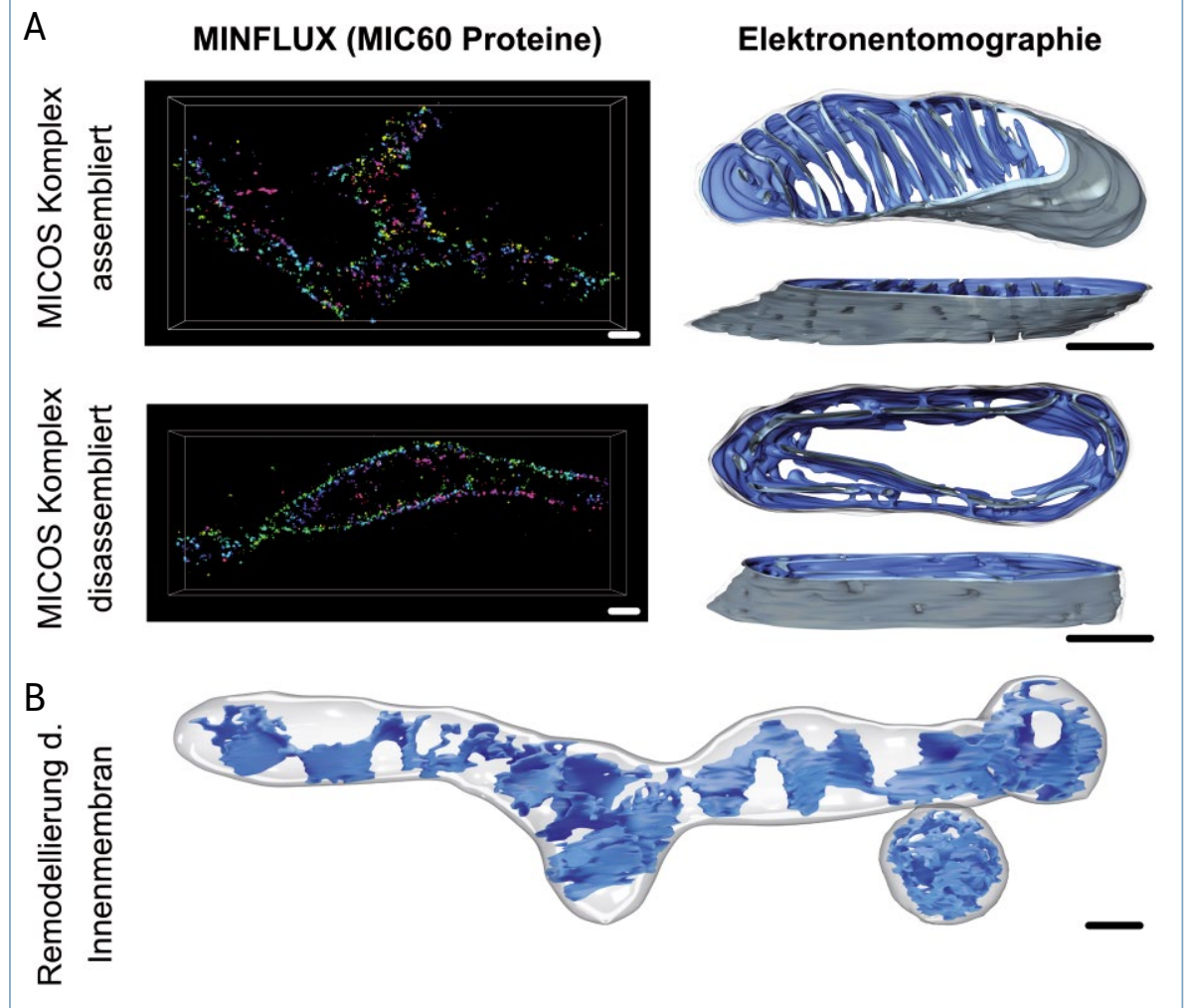

A Abb. 3: Einfluss des MICOS-Komplexes auf die Cristaearchitektur. A, 3D-MINFLUX-Nanoskopie des Crista-Junction-Markers MIC60 (links) und Rekonstruktionen der Innenmembran von Mitochondrien in Wildtypzellen und MIC 10-defizienten Zellen basierend auf Elektronentomographiedaten (rechts). B, Remodellierung der Cristae bei der Wiederherstellung des MICOS-Komplexes aus seinen beiden Teilkomplexen. Größenbalken: $250 \mathrm{~nm}$. Rekonstruktion der Innenmembran basierend auf FIB-SEM-Daten.

wieder her und verfolgten die Neubildung der MIC10-Teilkomplexe und ihre Assemblierung mit den noch bestehenden MIC60Teilkomplexen über einen Zeitraum von mehreren Stunden. Die damit einhergehenden Veränderungen der Membranstruktur konnten mithilfe von FIB-SEM analysiert werden. Es zeigte sich, dass die Bildung von MICOS zu einer fortschreitenden Reparatur der Cristaestruktur führte, bei der sich binnen weniger Stunden große, zuvor flachliegende Cristae wellenförmig aufstellten (Abb. 3B), um sich schließlich wieder in einzelne Lamellen umzuwandeln [8]. Diese Beobachtung zeigt, dass die Assemblierung von MICOS aus den beiden Teilkomplexen als molekularer Schalter fungiert, der die Trennung größerer Membranteile in einzelne Lamellen und die präzise Verteilung der Crista Junctions entlang der Mitochondrien steuert.

Die genaue Untersuchung dieses Prozesses erforderte den Einsatz zahlreicher unterschiedlicher molekulargenetischer, biochemischer und bildgebender Techniken. Das Beispiel unterstreicht, dass zunehmend komplexer werdende biologische Fragestellungen vielfach nur durch die Kombination der Stärken unterschiedlichster Analysetechniken gelöst werden können.

\section{Literatur}

[1] Giacomello M, Pyakurel A, Glytsou C et al. (2020) The cell biology of mitochondrial membrane dynamics. Nat Rev Mol Cell Biology 21: 204-224

[2] Klar TA, Jakobs S, Dyba M et al. (2000) Fluorescence microscopy with diffraction resolution barrier broken by stimulated emission. Proc Natl Acad Sci U S A 97: 8206-8210
[3] Stephan T, Roesch A, Riedel D et al. (2019) Live-cell STED nanoscopy of mitochondrial cristae. Sci Rep 9: 12419 [4] Kondadi AK, Anand R, Hänsch S et al. (2020) Cristae undergo continuous cycles of membrane remodelling in a MICOS-dependent manner. EMBO Rep 21: e49776 [5] Balzarotti F, Eilers Y, Gwosch KC et al. (2017) Nanometer resolution imaging and tracking of fluorescent molecules with minimal photon fluxes. Science 355: 606-612 [6] Gwosch KC, Pape JK, Balzarotti F et al. (2020) MINFLUX nanoscopy delivers 3D multicolor nanometer resolution in cells. Nat Methods 17: 217-224

[7] van der Laan M, Horvath SE, Pfanner N (2016) Mitochondrial contact site and cristae organizing system. Curr Opin Cell Biol 41: 33-42

[8] Stephan T, Brüser C, Deckers M et al. (2020) MICOS assembly controls mitochondrial inner membrane remodeling and crista junction redistribution to mediate cristae formation. EMBO J 39: e104105

Funding note: Open Access funding enabled and organized by Projekt DEAL. Open Access: Dieser Artikel wird unter der Creative Commons Namensnennung 4.0 International Lizenz veröffentlicht, welche die Nutzung, Vervielfältigung, Bearbeitung, Verbreitung und Wiedergabe in jeglichem Medium und Forma erlaubt, sofern Sie den/die ursprünglichen Autor(en) und die Quelle ordnungsgemäß nennen, einen Link zur Creative Commons Lizenz beifügen un angeben, ob Anderungen vorgenommen wurden. Die in diesem Artikel enthallenen Blder und sonstiges Ditin werial unterlingen ebenfalls der genannten Creative Commons Lizenz, sofen sich aus der Abbildungsleg genannten Creative Commons Lizenz steht und die betreffende Handlung nich nach gesetzlichen Vorschriften erlaubt ist, ist für die oben aufgeführten Weiterverwendungen des Materials die Einwilligung des jeweiligen Rechteinhabers einzuholen. Weitere Details zur Lizenz entnehmen Sie bitte der Lizenzinformation auf http://creativecommons.org/licenses/by/4.0/deed.de.

\section{Korrespondenzadresse:}

Prof. Dr. Stefan Jakobs

Universitätsmedizin Göttingen

Klinik für Neurologie

AG Hochauflösende Mikroskopie der Zelle

Robert-Koch-Straße 40

D-37075 Göttingen

sjakobs@gwdg.de

Max-Planck-Institut für biophysikalische Chemie Abt. NanoBiophotonik

AG Struktur und Dynamik von Mitochondrien Am Fassberg 11

D-37077 Göttingen

sjakobs@mpibpc.mpg.de

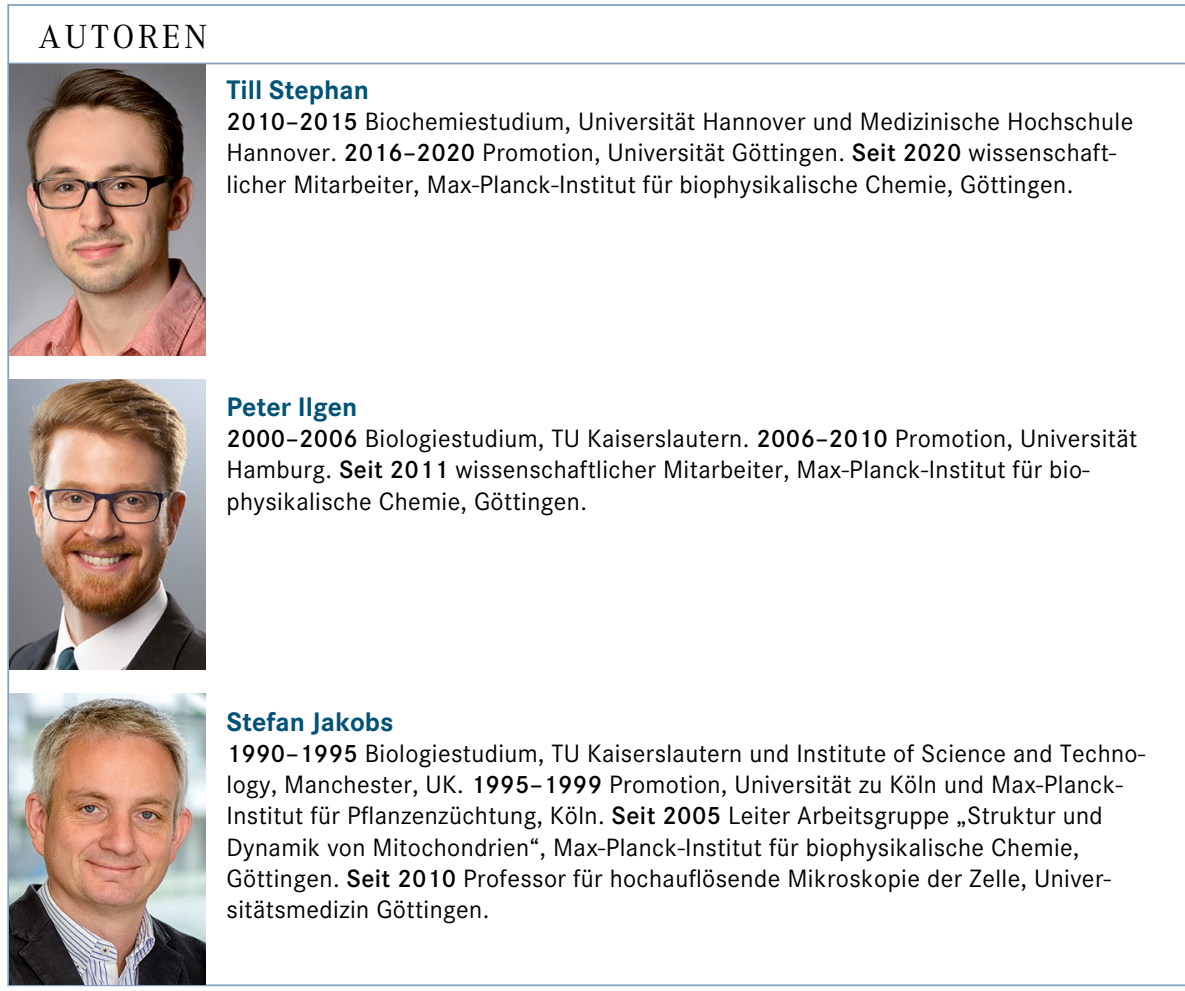

